\title{
LHCB RESULTS
}

\author{
Evgeny Gushchin ${ }^{a}$
}

Institute for nuclear research RAS, 117312 Moscow, Russia

Abstract. Experimental performance of $\mathrm{LHCb}$ detector with special emphasis on key measurements is presented. Also some recent results related to the search for New Physics are described.

\section{Introduction}

The Cabibbo-Kobayashi-Maskawa (CKM) matrix describes flavour mixing in the quark sector of the SM. The last decade was very rich with precise measurements of its elements mainly done at B-factories and Tevatron. Although modern experimental data are successfully described with the CKM mechanism, there are several tensions with observables such as $\operatorname{BR}(B \rightarrow \tau \nu), \sin (2 \beta)$, $A_{s l}$ and room remains for New Physics (NP), i.e. in $B_{d, s}$ mixing [1]. Also indirect searches for NP in loop-mediated processes suppressed in the SM can extend the discovery potential for new heavy particles to mass range far beyond that accessible in direct searches. The core LHCb physics program as presented in [5] concentrates on the precise measurement of the CKM angle $\gamma$ in $B \rightarrow D K$ tree decays and in loop-mediated charmless two-body B-decays, the measurement of $B_{s}$ mixing phase $\phi_{s}$, the helicity structure study in $B^{0} \rightarrow K^{*} \mu \mu$ decay, the determination of branching fraction of the rare decay $B_{s}^{0} \rightarrow \mu \mu$, and also studies of radiative $b \rightarrow s \gamma$ decays.

\section{Beauty production at LHC}

At the time of the conference the LHC was operating at a luminosity of about $2 \cdot 10^{33} \mathrm{~cm}^{-2} \cdot \mathrm{sec}^{-1}$ and energy of $\sqrt{s}=7 \mathrm{TeV}$, which are below the design values, but already larger then at Tevatron by 3 and 3.5 times correspondingly. LHCb operates at instant luminosities of $3-3.5 \cdot 10^{33} \mathrm{~cm}^{-2} \cdot \mathrm{sec}^{-1}$ in autoleveling mode, a value optimal for the selection and reconstruction of B-decays. The $p p \rightarrow b \bar{b}$ production cross-section at $\sqrt{s}=7 \mathrm{TeV}$ is about $300 \mu \mathrm{b}^{-1}[2]$ that is 3 times larger than at Tevatron and much larger than $1.2 \mathrm{nb}^{-1}$ of $b$ production cross-section at $Y(4 S)$ energy at B-factories. A sample of about $10^{12}$ of B-mesons can be produced in $\mathrm{LHCb}$ during a nominal year with all b-quark species present. For example, $B^{+}, B^{0}, B_{s}^{0}, \Lambda_{b}^{0}, B_{c}$ are produced in the proportion of about $40 \%: 40 \%: 10 \%: 10 \%: 0.1 \%$. But due to a large minimum-bias cross-section equal to $65 \mathrm{mb}^{-1}$ in $\mathrm{LHCb}$ acceptance only one of about $200 p p$ interactions contains $b$-quarks. This fact leads to the requirement of a very selective trigger based on the efficient event reconstruction and particle

\footnotetext{
${ }^{a}$ e-mail: guschin@inr.ru
} 
identification. It also should be mentioned that the charm production crosssection is about 20 times larger [3] then the $b$-quark one and therefore $\mathrm{LHCb}$ has a great potential for charm studies too.

\section{LHCb experiment}

The LHCb detector $[4,6]$ is a single-arm forward spectrometer designed for precise measurements of $\mathrm{CPV}$ and rare decays of $\mathrm{B}$-mesons in $p-p$ collisions at the LHC. To exploit the $b \bar{b}$ pair production at LHC peaked in the forward direction the LHCb acceptance covers pseudo-rapidity range of about $1.9<\eta<$ 4.9 , that allows to capture almost $40 \%$ of $b \bar{b}$ events within only about $4 \%$ of solid angle. The LHCb detector consists of a vertex locator (VELO), a tracking system with a warm magnet of $4 \mathrm{~T} \cdot \mathrm{m}$ integrated field, two RICH detectors, calorimeter and muon systems. A silicon microstrip detector called VELO surrounds the beam interaction point and provides a reconstruction of decay vertex with a precision of $\sigma_{x, y} \approx 13 \mu \mathrm{m}$ and $\sigma_{z} \approx 63 \mu \mathrm{m}$ for 25 decay tracks. The track momentum is measured with the precision of $\Delta p / p \approx 0.35-0.5 \%$ in a momentum range of $2-100 \mathrm{GeV} / \mathrm{c}$. Two RICH detectors allow charged kaon identification in a range of $2-100 \mathrm{GeV} / \mathrm{c}$ with typical efficiency of up to $90 \%$ while the fake rate from pions as kaons is kept below $10 \%$. The calorimeter system provides transverse energy measurement and identification of photons, electrons and hadrons for the zero-level trigger. Identification of muons is done by the muon system consisting of 5 detector planes interlaid with absorbers of about 20 interaction lengths. Muon identification efficiency reaches $97 \%$ with a misidentification rate of $1-2 \%$ for momenta above $10 \mathrm{GeV} / \mathrm{c}$. The excellent performance of $\mathrm{LHCb}$ detector was demonstrated already with the results obtained with the 2010 data. For example, the outstanding proper time resolution of about $50 \mathrm{fs}$ in VELO provided world-class measurements of the B-hadron lifetimes [7]. The quality of momentum measurements resulted in the world's best B-hadron mass measurements in various $B \rightarrow J / \psi(\mu \mu) X$ decays [8]. It is also well illustrated by the resolution of $J / \psi(\mu \mu)$ mass of $12.3 \mathrm{MeV} / \mathrm{c}^{2}$ [9], which is much better than in other LHC detectors.

\section{$4 \mathrm{LHCb}$ results}

The measurement of quarkonium production cross-sections at LHC energies in forward region is crucial for the understanding of the relative contributions of Color Octet and Color Singlet models. Also the measurements of exclusive dimuon events allow the study of the other QCD predicted states, such as the pomeron and odderon. The first measurements of differential production cross-sections $[9,10]$ of $J / \psi, \psi(2 S), Y$ done by LHCb at $\sqrt{s}=7 \mathrm{TeV}$ show good general agreement at high $p_{T}$ between data and theory predictions in 
particular with NLO NRQCD. However, to discriminate amongst the various models in prompt $J / \psi$ production the additional studies of other observables such as $J / \psi$ polarization will be necessary. The double $J / \psi$ production can be affected by the existence of charm tetra-quark states. The $\sigma^{J / \psi J / \psi}$ cross-section measured by LHCb using the first $35.2 \mathrm{pb}^{-1}$ of data [11] is in agreement with LO QCD calculation. The exclusive dimuon production has been measured [12] in resonant and non-resonant states through intermediate production of a $J / \psi$, $\psi(2 S)$ or $\chi_{c}$ state. The production of individual $\chi_{c 0}, \chi_{c 1}, \chi_{c 2}$ components is found to be roughly equal. LHCb has also reported results on the production of the $X(3872)$ [13], candidate to an exotic bound state.

The precise determination of the angle $\gamma$ of $B_{d}$ CKM Unitarity Triangle is one of major goals of the LHCb program. The comparison of its measurements in tree-level decays, such as $B \rightarrow D K$ and in loop-mediated processes, such as $B^{0} \rightarrow \pi^{+} \pi^{-}$can provide a sign of NP. The data sample collected by LHCb until now is too small to allow the measurement of the $\gamma$-angle, but several studies have been done for validation of this program. With the $320 \mathrm{pb}^{-1}$ of data collected in $2011 \mathrm{LHCb}$ reported the most precise single measurement of direct CPV in $B_{d}^{0} \rightarrow K \pi$ and the first evidence of CPV in $B_{s}^{0} \rightarrow K \pi$ decay [14]. Another important search for open-charm $B^{ \pm} \rightarrow\left(K^{\mp} \pi^{ \pm}\right)_{D} K^{ \pm}$decay with 343 $\mathrm{pb}^{-1}$ of data has been reported by LHCb in [15]. The evidence of this mode is seen with significance of $4.0 \sigma$. Also the measurements of the observables $R_{A D S}$ and $A_{A D S}$ that relate to an extraction of the weak phase $\gamma$ have been obtained. With the $2 \mathrm{fb}^{-1}$ of integrated luminosity to be collected at the end of $2012 \mathrm{LHCb}$ expects to measure the angle $\gamma$ with a precision of about $5^{\circ}$ [5].

The rare decay $B^{0} \rightarrow K^{*} \mu^{+} \mu^{-}$is considered as highly sensitive to the contribution of NP changing its helicity structure. The most prominent observable is the forward-backward asymmetry of the muon system $A_{F B}\left(q^{2}\right)$ varying with the invariant mass of the dimuon pair. The zero-crossing point of $A_{F B}\left(q^{2}\right)$ is well defined in the SM, where the leading hadronic uncertainties cancel. The present analysis is based on the $309 \mathrm{pb}^{-1}$ of data taken during 2011 [16]. There is a good agreement observed between the SM prediction and the LHCb measurement of $A_{F B}$, which is currently the most precise world's measurement.

The ability to resolve the very fast $B_{s}^{0}$-oscillations is critical to the measurements of CPV in $B_{s}^{0}$ system. LHCb has reported the world's best measurement of the oscillation frequency parameter $m_{s}=17.63 \pm 0.11$ (stat.) \pm 0.04 (syst.) $\mathrm{ps}^{-1}$ [19]. The decay $B_{s}^{0} \rightarrow J / \psi \phi$ is considered as a golden mode for measurement of CPV phase in $B_{s}^{0}$ mixing. In SM the value of the phase is well predicted to be $\phi_{s} \cong-2 \beta_{s}$. Its value is estimated from global fit as $2 \beta_{s} \simeq 0.0363_{-0.0015}^{+0.0016}$ rad [20]. The measurement of the contribution of NP to the phase $\phi_{s}$ requires high precision. Experiments on Tevatron attained precision of measurement of $0.5 \mathrm{rad}$ [21]. Using the statistics of 2011 year LHCb can reach an accuracy of $0.1 \mathrm{rad}$. With data recorded in $2010 \mathrm{LHCb}$ obtained the result as a confidence 
region in $\phi_{s}-\Delta \Gamma_{s}$ plane with deviation from SM point at $1.2 \sigma$ level [22].

The search for the rare decay $B_{s}^{0} \rightarrow \mu^{+} \mu^{-}$is one of the most promising ways to test the SM. The branching ratio (BR) of this decay is computed [17] to be $\operatorname{BR}\left(B_{s}^{0} \rightarrow \mu^{+} \mu^{-}\right)=(3.2 \pm 0.2) \cdot 10^{-9}$. The upper limit measured by LHCb using $300 \mathrm{pb}^{-1}$ of data is $\operatorname{BR}\left(B_{s}^{0} \rightarrow \mu^{+} \mu^{-}\right)<1.3(1.6) \cdot 10^{-8}$ at $90 \%(95) \%$ CL [18] that is less than factor 5 from the SM prediction.

\section{Conclusion}

The large $b$-quark production rate at LHC and excellent performance of LHCb detector creates a great opportunity for precise measurements of CPV and rare decays with B-mesons. All necessary components like mass and proper time resolution, particle identification and tagging capabilities, have been proved with data taken in 2010 and 2011 runs. The results obtained with key channels of the core physics program confirm the LHCb potential for the search for NP.

\section{References}

[1] A. Lenz et al., Phys.Rev. D 83, 036004 (2011).

[2] The LHCb Collaboration, Phys. Let. B 694,209-216, (2010).

[3] The LHCb Collaboration, CERN-LHCb-CONF-2010-013.

[4] LHCb Technical Design Report, CERN/LHCC 2003-030, (2003).

[5] The LHCb Collaboration, arxiv:0912.4179v2 [hep-ex].

[6] The LHCb Collaboration, JINST 3 (2008) S08005.

[7] The LHCb Collaboration, CERN-LHCb-CONF-2011-001.

[8] The LHCb Collaboration, CERN-LHCb-CONF-2011-027.

[9] The LHCb Collaboration, Eur. Phys. J. C 71, 1645, (2011).

[10] The LHCb Collaboration, CERN-LHCb-CONF-2011-026; CERN-LHCbCONF-2011-016.

[11] The LHCb Collaboration, CERN-LHCb-CONF-2011-009.

[12] The LHCb Collaboration, CERN-LHCb-CONF-2011-022.

[13] The LHCb Collaboration, CERN-LHCb-CONF-2011-021.

[14] The LHCb Collaboration, CERN-LHCb-CONF-2011-042.

[15] The LHCb Collaboration, CERN-LHCb-CONF-2011-044.

[16] The LHCb Collaboration, CERN-LHCb-CONF-2011-038.

[17] A.J. Buras, arXiv:1012.144\%; A.J. Buras et al., arXiv:1007.5291.

[18] The LHCb Collaboration, CERN-LHCb-CONF-2011-037.

[19] The LHCb Collaboration, CERN-LHCb-CONF-2011-005.

[20] J. Charles et al., Eur. Phys. J. C41 ,1-131, (2005), hep-ph/0406184

[21] The CDF Collaboration, CDF/ANAL/BOTTOM/PUBLIC/10206 (2010); The D0 Collaboration, D0 Conference note 6098-CONF (2010).

[22] The LHCb Collaboration, CERN-LHCb-CONF-2011-006. 